\title{
LATE COMERS ATTENDANCE MONITORING AND SMS ALERT SYSTEM
}

\author{
Rudra Pratap Das \\ Professor, R\&D Director \\ NSRIT, Visakhapatnam, India \\ Rokkam Tharun Babu, Sabbavarapu Mohini, Kotyada Lokesh, Puttupu Sasikanth \\ Student, ECE \\ NSRIT, Visakhapatnam, India
}

\begin{abstract}
In any institution/offices, Managing the records of attendance of the staff and students or employees is a tedious task. Our concept is to take attendance for the late coming persons in the colleges and sending SMS to their respective parents/ guardians. This task can be made by using biometric process or by image processing. so, for the simplified task we use biometric system.
\end{abstract}

Keywords-Node MCU, Fingerprint Sensor, OLED Display, Xampp Web server.

\section{INTRODUCTION}

we use fingerprint sensor to record the fingerprints of students and we take attendance for the late coming students, the data of the late comers will be auto generated and stored in a excel sheet through the web-server after receiving the excel sheet, daily we can send SMS through that excel sheet by using a inbuilt SMS Excel plug-in. If a Student comes late continuously for 3 days then automatically another Warning SMS will be send to that particular parent regarding of his/her ward coming late and if the continued to be late then Fine will be charged for that particular student. This sending of SMS can be done by using a SMS Excel plug-in. In order to update our process with respect to time and to maintain the database of the late comers students daily we are using a web-server. Here we are using fuzzy logic to identify the correct person through Finger print module because, Identifying some amount of people through the finger print can be done but when the Fingerprint enrolling data increases we can't identify them. So for this purpose fuzzy logic is used here In any Attendance system to record the data fast and simple biometric Fields are used, we are having various forms to manage biometric systems likely RFID system, Fingerprint system, Face recognition and IRIS scan system. When comparing to each and every process, Fingerprint recognition is having more accurate results and faster response. Fingerprints are one of the main forms in biometric field which used to identify the individual and their uniqueness. Fingerprint authentication is one of popular authentication systems in the world. Due to their consistency and uniqueness it is used widely. There are several sources available for the collection and their established use. This paper presents the attendance management system using fingerprint technology in a university environment. It consists of two processes that is enrolment and authentication. During the enrolment process we need to take individual persons identity that is their fingerprints and stores them in a EEPROM of the Finger-print Scanner. We are using Fingerprint scanner of model R307 which can store the Fingerprints up to 127 . So we can Store 127 individual persons Fingerprints. To maintain the records of Students attendance a local host web server is used by using PHP, java script and CSS languages and by this, we can directly convert the daily users log into a excel sheet.

\section{HARDWARE REQUIREMENTS}

\section{Node MCU}

NodeMCU V3 is an open-source firmware and development kit that plays a vital role in designing an IoT product using a few script lines. Multiple GPIO pins on the board allow us to connect the board with other peripherals and are capable of generating PWM, I2C, SPI, and UART serial communications.

The interface of the module is mainly divided into two parts including both Firmware and Hardware where former runs on the ESP8266 Wi-Fi SoC and later is based on the ESP-12 module.

The firmware is based on Lua - A scripting language that is easy to learn, giving a simple programming environment layered with a fast scripting language that connects you with a well-known developer community the existing module and keep changing the entire interface until you succeed in optimizing the module as per your requirements. to UART data which mainly understands the language of serial communication. 


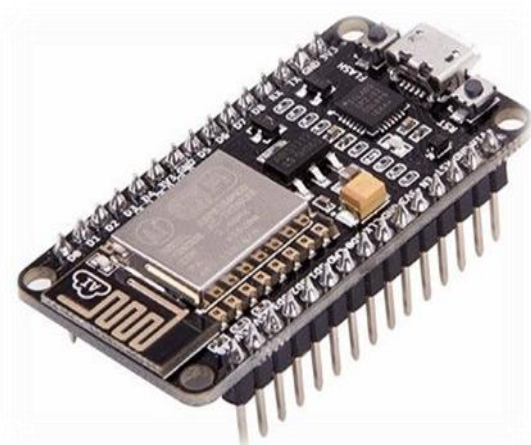

Fig 1. Node MCU Board

\section{Fingerprint Sensor R307}

This is the R307 Optical Fingerprint Reader Sensor Module. R307 fingerprint module is a fingerprint sensor with a TTL UART interface for direct connections to microcontroller UART or to PC through MAX232 / USB-Serial adapter. The user can store the fingerprint data in the module and can configure it in 1:1 or 1: $\mathrm{N}$ mode for identifying the person.

The FP module can directly interface with 3.3 or $5 \mathrm{v}$ Microcontroller. A level converter (like MAX232) is required for interfacing with $\mathrm{PC}$ serial port.

Integrated image collecting and algorithm chip together, ALLin-One Fingerprint reader can conduct secondary development, can be embedded into a variety of end products. Users can conduct secondary development, can be embedded into a variety of end products, such as access control, attendance, safety deposit box, car door locks

Low power consumption, low cost, small size, excellent performance, Professional optical technology, precise module manufacturing techniques. Good image processing capability can successfully capture an image up to resolution 500 dpi Finger detection function.
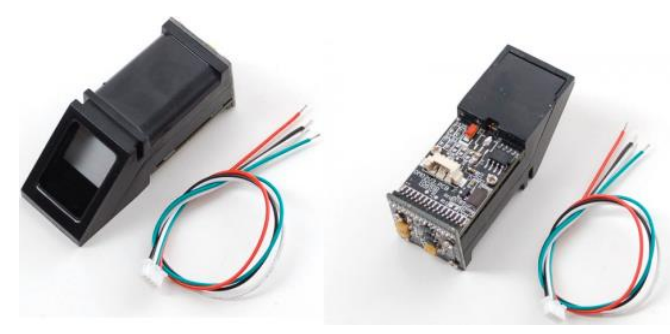

Fig 2. Fingerprint Sensor

\section{OLED Display}

0.96 inch OLED Display Module is a precise small, White OLED module which can be interfaced with any microcontroller using SPI protocol. It is having a resolution of 128x64. The package includes display board, display, 6 pin male header.

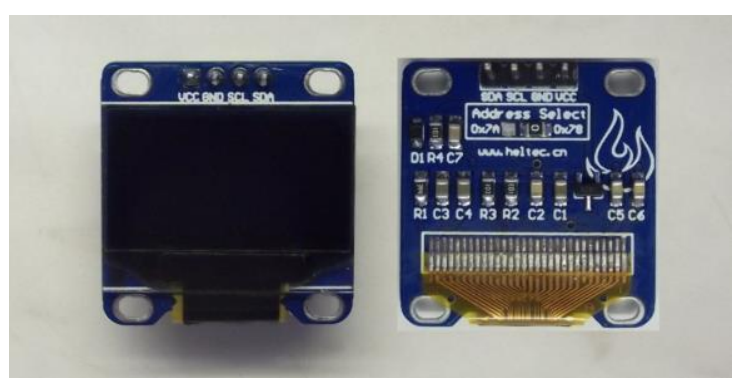

Fig 3:- OLED Display

OLED (Organic Light-Emitting Diode) is a self light-emitting technology composed of a thin, multi-layered organic film placed between an anode and cathode. In contrast to LCD technology, OLED does not require a backlight. OLED possesses high application potential for virtually all types of displays and is regarded as the ultimate technology for the next generation of flat-panel displays.

\section{SMS EXCEL PLUGIN}

An add-in is software that adds new features into Microsoft Excel.Add-ins save you time. Using an add-in helps to avoid errors and do repetitious work in minutes that could take hours manually. The Spreadsheet Assistant adds over 200 time saving features to Microsoft Excel.

SMS Excel Plugin offers users to send SMS from Excel sheet directly. As most Business Professionals use MS Excel for organizing their customer data, along with its feature to send SMS from Excel, empowers all users to save their precious time and send personalized bulk SMS.

SMS Excel Plugin can be directly integrated with Microsoft Excel and one can send different SMS to different mobile number at once instantly.

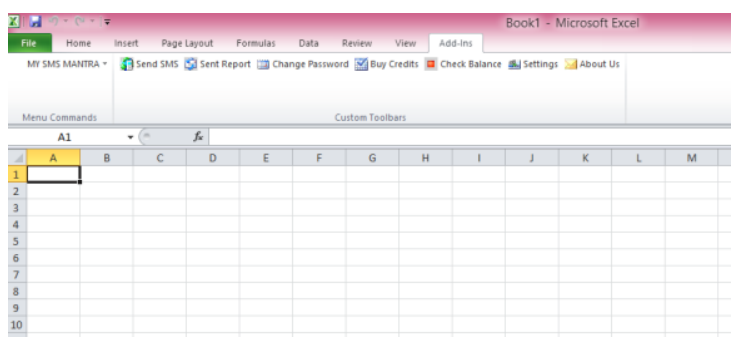

Fig:- 4 Excel SMS Add-in

There are many SMS Excel services in internet, where as we are using MY SMS MANTRA service to send the SMS.

This bulk SMS software can be used to propagate the business updates. My SMS Mantra is a free bulk SMS software allowing users to schedule messages with multilingual support. The automated messaging process provides reporting details also. 


\section{XAMPP WEB SERVER}

XAMPP is a free and open-source cross-platform web server solution stack package developed by Apache Friends, consisting mainly of the Apache HTTP Server, Maria DB database, and interpreters for scripts written in the PHP and Perl. Since most actual web server deployments use the same components as XAMPP, it makes transitioning from a local test server to a live server possible.

XAMPP's ease of deployment means a WAMP or LAMP stack can be installed quickly and simply on an operating system by a developer, with the advantage that common addin applications such as WordPress and Joomla can also be installed with similar ease using Bitnami.

Apache: ihe open source web server Apache is the most widely used server worldwide for delivery of web content. The server application is made available as a free software by the Apache Software Foundation.

MySQL/MariaDB: in MySQL, XAMPP contains one of the most popular relational database management systems in the world. In combination with the web server Apache and the scripting language PHP, MySQL offers data storage for web services. Current XAMPP versions have replaced MySQL with MariaDB (a community-developed fork of the MySQL project, made by the original developers).

PHP: the server-side programming language PHP enables users to create dynamic websites or applications. PHP can be installed on all platforms and supports a number of diverse database systems.

Perl: the scripting language Perl is used in system administration, web development, and network programming.

\section{Block Diagram}

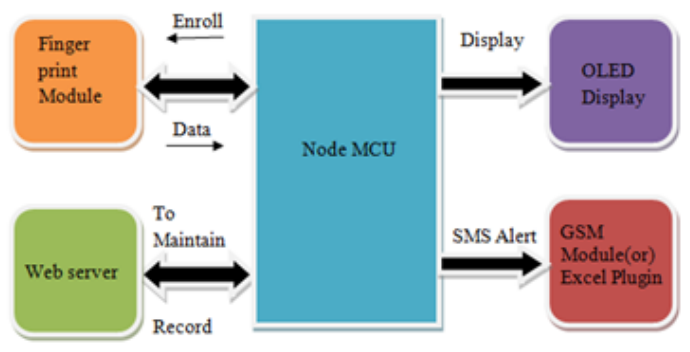

Fig :- 5 Block diagram

From the above figure 5 we can know the block diagram of Late comers attendance monitoring and SMS alert system. It consists of two sections i.e; Input section and Output section. Input section Consists of Finger print module to Enroll, Delete, Store the data of Finger prints. Web server is used for the purpose of storing the Finger print enrolled data and to maintain a data base by which we can see the data of finger prints whenever we need.

Output section consists of OLED display and Excel plugin .To display the correct identification of the persons like Names, Finger print symbol and etc we use OLED display here. Excel plugin is used to Send SMS to parents directly through Excel sheet without interfering any GSM module.

Another section which plays a crucial part in the block diagram is Controller Section. We are using Node MCU as a Controller part, All the input sections and Output sections are connected to this Node MCU. First it takes input finger prints from users to enroll their data, after enrolling Fingerprints the device will be ready then it sends the entered fingerprint data to Web server continuously to update it with respect to time, At the same time it will sends the data to OLED display for the purpose of displaying the users data, after the users complete their process of attendance through the web server's website we can convert the direct data into a Excel sheet. By downloading the excel sheet we can maintain a record of persons then excel plugin is used to convey the message at a time to all the registered users.

\section{SOFTWARE}

The Arduino IDE Integrated Development Environment is a cross-platform application written in Embedded C, and is derived from the IDE for the Processing programming language and the Wiring projects. It includes a code editor with features such as syntax high-lighting, braces matching and automatic indentation, and is also capable of compiling and uploading programs to the board with a single click. A program or code written for Arduino is called a "sketch". Arduino programs are written in $\mathrm{C}$ or $\mathrm{C}++$.

\section{REsUlts}

Results are shown in a box in which it contains fingerprint module, Oled display and Node MCU inside of it.

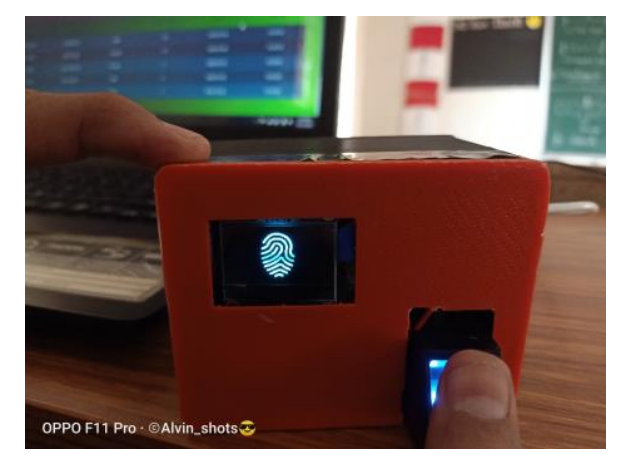

Fig:- 6 Experimental results 


\section{International Journal of Engineering Applied Sciences and Technology, 2020 \\ Vol. 4, Issue 11, ISSN No. 2455-2143, Pages 259-262 \\ Published Online March 2020 in IJEAST (http://www.ijeast.com)}

\section{CONCLUSION}

In this project, we have presented a Late Comers Attendance Monitoring and SMS Alert System which is a fingerprintbased attendance management system. The developed system is an embedded system that is part of a fingerprint recognition/authentication system based on minutiae points. The system extract the local characteristic of a fingerprint which is minutiae points in template based. Templates are matched during both registration and verification processes. For improved quality control during the registration or verification process, a matching score was used to determine the success of the operation.

\section{FUTURE SCOPE}

In future we would like to add in-built SMS Alert System that can be done by using GSM Module, then we can send SMS directly to their parents without interfering with any other software. But the problem occurs of using GSM Module is to be in a good active signal.

\section{REFERENCES}

[1] K.Jaikumar, M.santhosh Kumar, 2015, Fingerprint based attendance system with SMS Alert to parents, IJRET, Volume: 04 Issue: 02, Feb-2015, pg:293-295.

[2] O.Shoewu and O.A Idowu , 2012, Development of Attendance management system using Biometrics, The pacific Journal of Science and Technology, pg:300-307.

[3] C.O Akinduyte, A.O Adetum, 2013, Fingerprint based attendance management system by - Journal of Computer science and applications.

[4] karthik krishnamurthi, S.Irudaya Mary, 2015, Fingerprint Based attendance system, IJARCCE, DOI 10.17148, pg:621623

[5] L.S. Ezema, C.K.A Joe-Uzuegbu, 2015, Fingerprint based attendance management system, IJSER.

[6] J.Sangeetha, S.Sivaranjani, 2015, Fingerprint based attendance management system, IJEDR

[7] Hitesh Walia1, Neelu Jain, May-2016, Fingerprint based attendance systems-A Review, IRJET, Volume:03 Issue:05.

[8] B. K. P. Mohamed and C. V. Raghu, 2012, "Fingerprint attendance system for classroom needs," 2012 Аnnual IEEE India Conference (INDICON), pp. 433-438.

[9] Seng Chun Hoo and Haidi Ibrahim, 2019, Biometric-Based attendance tracking system for education sectors, Hindawi Journal of sensors, pg:14-17.
[10] Pallavi Verma and Namit Gupta, 2013, Fingerprint based student attendance system using GSM, IJSR Volume 2 Issue 10, October 2013, pg: 128-131.

[11] Khin San Myint, Chan Mya Mya Nyein, 2018, Fingerprint based attendance system using arduino, International Journal of Scientific and Research Publications, Volume 8, Issue 7, July 2018, pg: 422-426.

[12] J.Ram Prabu , R.Pavithra, 2018, Wireless Smart biometric attendance system, International Journal of Innovative Technology and Exploring Engineering (IJITEE), Volume-8 Issue-2S December, 2018, pg: 156-159. 